\title{
Apoptosis and Its Significance in Oral Diseases: An Update
}

\author{
Megha Jain, ${ }^{1}$ Sowmya Kasetty, ${ }^{2}$ Sudheendra Udyavara Sridhara, ${ }^{3}$ Nitin Jain, ${ }^{4}$ \\ Samar Khan, ${ }^{5}$ and Ami Desai ${ }^{2}$ \\ ${ }^{1}$ Department of Oral Pathology and Microbiology, People's Dental Academy, MIG, Block C, Flat No. 14, PCMS Campus, \\ People's Hospital, Bhopal, Madhya Pradesh 462037, India \\ ${ }^{2}$ Department of Oral Pathology and Microbiology, People's College of Dental Sciences and Research Center, Bhopal, \\ Madhya Pradesh 462037, India \\ ${ }^{3}$ Department of Oral Pathology and Microbiology, Coorg Institute of Dental Sciences, Virajpet, Karnataka 571218, India \\ ${ }^{4}$ Department of Otolaryngology, All India Institute of Medical Sciences (AIIMS), Raipur, Chhattisgarh 492099, India \\ ${ }^{5}$ Department of Oral Pathology and Microbiology, Rishiraj Dental College, Bhopal, Madhya Pradesh 462036, India
}

Correspondence should be addressed to Megha Jain; megha.vipin12@gmail.com

Received 24 September 2013; Accepted 8 December 2013

Academic Editor: Mitsuhiro Ohshima

Copyright (C) 2013 Megha Jain et al. This is an open access article distributed under the Creative Commons Attribution License, which permits unrestricted use, distribution, and reproduction in any medium, provided the original work is properly cited.

Apoptosis is a well defined mode of cell death which plays an imperative role in the development, regulation, and maintenance of the cell populations in multicellular organisms. Apoptosis is implicated in both health and diseases. Errors in apoptotic mechanisms have been allied to a wide range of pathologies including oral diseases. This review presents an update focused on the role and significance of apoptosis in various oral diseases ranging from reactive to benign and malignant pathologies.

\section{Introduction}

The word "apoptosis" is of Greek origin, meaning "dropping off" or "falling off" of petals from flowers or leaves from trees. The term "apoptosis" was first introduced by Kerr et al. It is an active, inherently programmed phenomenon which can be initiated or inhibited by a variety of environmental stimuli, both physiological and pathological. Carl Vogt, however, first described the phenomenon more than 100 years earlier in $1842[1,2]$.

There are four major players which are involved in triggering and influencing the apoptotic process. These are the caspases, Bcl-2 family of proteins, tumor necrosis factor receptor (TNF-R) superfamily, and adaptor proteins [3]. Caspases are a family of proteases and are classified into initiator caspases 2, 8, 9, and 10, effector caspases 3,6, and 7, and inflammatory caspases $1,4,5,11,12,13$, and 14 [3-5]. The members of Bcl-2 family are a group of crucial regulatory factors in apoptosis which are characterized by presence of conserved sequence motifs known as Bcl-2 homology (BH) domains. They are categorised into proapoptotic proteins (Bad, Bak, Bax, Bcl-rambo, Bcl-xS, Bid, Bik, Bim, Blk, BNIP3, Bok/Mtd, Hrk, and Nip3 in mammals and Egl-1 in C. elegans) and antiapoptotic proteins (A1/Bfl1, Bcl-2, Bcl-w, Bcl-xL, Boo/Diva, Mcl-1, NR-13, and Nrf3 in mammals, BHRF-1, E1B19 K, Ks-Bcl-2, LMW5-HL, and ORF16 in bacteria, and Ced-9 in C. elegans) $[3,5]$.

Broadly based intrinsic and extrinsic classification is used as platform to describe apoptotic signal cascade. Extrinsic/death receptor activated pathway involves the initiation of apoptosis through ligation of plasma membrane death receptors whereas intrinsic/mitochondrial cell death pathway involves initiation of apoptosis as a result of disturbance of intracellular homeostasis and mitochondria being critical executioner of cell death. While the initiation mechanisms of these pathway are different, both pathways converge (common pathway) resulting ultimately in cellular morphologic and biochemical alterations characteristic of apoptosis $[5,6]$. Thus, apoptosis is a tightly synchronised and efficient cell death program requiring interplay of multitude of factors.

Apoptosis is a genetically programmed form of cell death which is indispensable for development and homeostasis of multicellular organism. Although apoptotic cell death plays an important role in maintenance of the normal physiological state, it may also be responsible for diseased 
state of the body. A dysfunctional apoptotic system can lead to either excessive removal or prolonged survival of cells and thus dysregulated apoptosis is implicated in pathogenesis of a variety of diseases including oral pathologies. The present review attempts to summarise and highlight the role and significance of apoptosis in various oral diseases.

\section{Classification of Oral Diseases}

(1) Reactive lesions

(i) Pyogenic granuloma

(ii) Giant cell granuloma

(2) Inflammatory diseases

(i) Recurrent aphthous stomatitis (may fall into more than one category)

(ii) Periodontal diseases

(3) Immune-mediated diseases

(i) Oral lichen planus

(ii) Erythema multiforme

(iii) Lupus erythematosus

(iv) Pemphigus vulgaris

(v) Epidermolysis bullosa

(vi) Oral graft-versus-host disease

(vii) Sjögren's syndrome

(4) Virus associated diseases

(i) HSV associated

(ii) HPV associated

(iii) HIV associated

(5) Benign entities

(i) Benign odontogenic cysts and tumors
(a) Radicular cyst
(b) Dentigerous cyst
(c) Odontogenic keratocyst/keratocystic odon- togenic tumor
(d) Ameloblastomas

(ii) Benign salivary gland tumors

(iii) Benign epithelial tumor

(a) Squamous papilloma

(6) Premalignant and malignant entities
(i) Actinic cheilitis
(ii) Oral leukoplakia
(iii) Oral squamous cell carcinoma
(iv) Malignant salivary gland tumors.

\section{Apoptosis in Oral Diseases}

\subsection{Apoptosis and Reactive Lesions}

3.1.1. Apoptosis and Pyogenic Granuloma. Cells in multicellular organisms are inflicted with a variety of challenges to which they react by regeneration, hyperplasia, dysplasia, hypertrophy, atrophy, or metaplasia depending on the nature of the challenge. These modes of cellular adaptability involve modulation of the balance between cell proliferation and cell death [7]. Pyogenic granuloma (PG) is one of the inflammatory hyperplasias seen in the oral cavity which may arise in response to various stimuli such as low-grade local irritation, traumatic injury, or hormonal factors [8]. Lower ISTR (in situ 3 -tailing reaction) labeling indices and more frequent $\mathrm{Bcl}$ 2/Bax expression in PG than granulation tissue and capillary hemangioma suggested that the low apoptotic rate in PG is closely related to its characteristic rapid growth [9]. It has also been found that, in pregnancy tumor, female sex hormones not only enhance the expression of angiogenic factors but also decrease apoptosis of granuloma cells to extend angiogenic effect [10]. VEGF alone or in combination with Ang-2 could protect microvessels from apoptosis, while Ang-2 alone had no effect. Therefore lack of VEGF is associated with apoptosis of endothelial cells and regression of granuloma [11].

3.1.2. Apoptosis and Giant Cell Granuloma. Central giant cell lesion (CGCL) and peripheral giant cell lesion (PGCL) of the jaws are characterized by multinucleated osteoclast-like giant cells in a background of mononuclear cells [12, 13]. Pammer et al. [13] demonstrated strong expression of Bax and Bak, weak positivity for $\mathrm{Bcl}-2$, and moderate positivity for Bcl-x in majority of giant cells in PGCL and lesions containing osteoclast-like giant cells, whereas only scattered mononuclear cells were positive for Bax, Bak, and Bcl-2. Also, frequency of apoptotic nuclei was 18 times higher in giant cells than in mononuclear cells suggesting that giant cells of bone and soft tissue tumors are reactive cell forms and are not of neoplastic origin. Both CGCL and PGCL showed increased expression of Bax and Bcl-2 mRNA. PGCL showed a higher apoptotic index (ratio $\mathrm{Bax} / \mathrm{Bcl}-2$ ) than CGCL, also confirmed by TUNEL analysis which might be associated with the different clinical behavior of CGCL and PGCL [12].

\subsection{Apoptosis and Inflammatory Diseases}

3.2.1. Apoptosis and Recurrent Aphthous Ulceration. Recurrent aphthous ulceration (RAU) is a common inflammatory condition of the oral mucosa predominantly affecting lining oral mucosa [7]. Recurrent aphthous like ulceration is a cardinal feature of Behcet's syndrome and is consistently associated with it [14]. Ultrastructurally, normal oral mucosa of patients with RAU or Behcet's syndrome revealed degenerated, apoptotic prickle cells and it was speculated that the onset of aphthous ulceration is closely related to phagocytosis of these apoptotic cells by intraepithelial mononuclear cells [15]. RAU induces p53 immunoexpression which might be related to its aetiopathogenesis [16]. 
3.2.2. Apoptosis and Diseases of Periodontium. Periodontal disease is an acute or chronic inflammatory condition which develops as a result of imbalance in the equilibrium between the host defence and microbial agents resulting in destruction of tooth-supporting tissue leading to mobility and tooth loss. TUNEL positive cells, positive staining with active caspase3, Fas, FasL, p53, and chromatin condensation was found in the inflammatory infiltrates of periodontitis patients whereas Bcl-2 positivity was found in both periodontitis patients and healthy controls, thus concluding that apoptotic mechanism could be implicated in the inflammatory process associated with periodontitis [17]. A greater number of apoptotic keratinocytes than proliferative ones are found in periodontitis patients, only in the most apical part of the sulcus compared to those with gingivitis [18]. Monocytes are the most lysis-sensitive leukocytes for A. actinomycetemcomitans leukotoxin, which depends on caspase 1-activation [19]. It is also evidenced that live $P$. gingivalis can induce significant apoptosis in primary human gingival epithelial cells between 12 and 24 hours of challenge via gingipains dependent mechanism [20]. Hydrogen sulfide can induce apoptosis in gingival epithelial cells and human gingival fibroblast in the oral cavity which may cause a periodontal condition [21]. Neutrophils, when combined in a $P$. gingivalis challenge assay of epithelial cells, prevent epithelial cell apoptosis by phagocytosing $P$. gingivalis and also protect the host from the harmful effects of its microbial proteases [22]. It has also been analysed that the increased fibroblast proliferation and a simultaneous decrease in apoptosis contribute to gingival overgrowth [23].

\subsection{Apoptosis and Immune-Mediated Diseases}

3.3.1. Apoptosis and Oral Lichen Planus. The hallmark histopathologic feature of lichen planus (LP) is the degeneration of the basal layer of the epithelium. Eosinophilic globular bodies also known as colloid, hyaline, cytoid, or Civatte bodies are also characteristic features of LP, seen in the basal layer and upper connective tissue, and have been interpreted as degenerative keratinocytes. Damage to the basal layer appears to involve apoptosis as an early event [24]. The following mechanisms are proposed for keratinocyte apoptosis:

(1) T-cell-secreted TNF- $\alpha$ binding to TNF- $\alpha$ R1 receptor on keratinocyte surface,

(2) T-cell surface CD95L (Fas ligand) binding to CD95 (Fas) on the keratinocyte surface,

(3) T-cell-secreted granzyme B entering the keratinocyte via perforin induced membrane pores.

All these mechanisms activate caspase cascade resulting in keratinocyte apoptosis. On the contrary reduced or absent apoptotic rate in inflammatory cells has been thought to contribute to development of OLP [25].

One of the studies demonstrated that caspases 2 and 12 were expressed in all samples and caspase- 8 was expressed in most of the samples of atrophic lichen planus suggesting the importance of endoplasmic reticulum (ER) stress in inducing apoptosis in OLPs. However, the activation of death receptors in OLP cannot be ruled out [26]. Increased apoptosis may cause a decrease in epithelial thickness reflected in the activity of the lesion. It was found that apoptosis in the erosive OLP was significantly more intense than in the reticular type and both forms of OLP presented more apoptosis than the healthy oral mucosa [27]. It has been demonstrated that increased numbers of apoptosis were detected in OLP, especially in association with lymphocytic infiltration and hematoxylin-eosin staining being more sensitive in detecting higher number of apoptotic nuclei than in situ end labeling [28]. Expression of cell proliferation proteins like PCNA and apoptotic proteins like Bcl-2 and Bax was found to be altered in OLP as seen in epithelial dysplasia suggesting their potential for malignant transformation [29]. TUNEL and caspase- 3 expression showed absence or low rate of apoptosis in inflammatory cells in OLP thus contributing to the persistence of the inflammation and favouring cancer development [30]. Apoptosis in OLP seems to be correlated with granzyme B release, while in oral cGVHD perforin seems to be more important suggesting that subtle differences seem to exist in their pathogenetic mechanisms [31].

3.3.2. Apoptosis and Erythema Multiforme. Erythema multiforme is a distinct dermatologic hypersensitivity pathology characterized by cutaneous or mucous lesions and eventually it can also involve both. In their more severe forms, they appear with occasional visceral involvements. It includes wide range of clinical patterns-EM minor, EM major, Stevens Johnson syndrome, and toxic epidermal necrolysis [32]. Cell death in EM is at least in part due to apoptosis which may be related to an altered expression of apoptosisregulating proteins like $\mathrm{p} 53$ and $\mathrm{Bcl}-2$ family proteins and activation of Fas/Fas-ligand system [33]. ELISA and in vitro analysis showed that sFasL secreted by PBMCs not keratinocytes plays a crucial role in the keratinocyte apoptosis and pathogenetic mechanism of TEN and SJS and serum sFasL level may be a good indicator for the early diagnosis of TEN and SJS [34]. It has also been found that perforin mediates apoptosis in the pathogenesis of the epidermal cell changes in SJS [35].

3.3.3. Apoptosis and Lupus Erythematosus. Systemic lupus erythematosus (SLE) and discoid lupus erythematosus (DLE) are autoimmune diseases manifesting as erythematous or ulcerative lesions with white striae radiating from the margins [7]. CD95 mutations are linked with loss of regulation of B lymphocytes, which predisposes to systemic autoimmunity including SLE [36]. Autoantigens in SLE become clustered in and on the surface blebs of apoptotic cells and these cells play a central role in tolerizing $\mathrm{B}$ cells and $\mathrm{T}$ cells and may drive the autoimmune response in systemic autoimmune disease [37]. SLE is characterized by imbalance redox state which in turn is related to intracellular levels of glutathione and controlled production of reactive oxygen species and alteration in the intracellular redox environment of cells are critical for cellular immune dysfunction, activation of apoptotic enzymes, and apoptosis [38]. It has also been demonstrated that elevated endothelial cell apoptosis strongly correlates with markedly 
abnormal vascular function and elevated tissue factor levels and may represent an important mechanism for development of atherothrombosis in SLE [39].

3.3.4. Apoptosis and Pemphigus Vulgaris. Pemphigus is a group of autoimmune blistering diseases characterized by loss of keratinocyte cell adhesion that leads to clinical blister formation. Induction of apoptosis or of proapoptotic proteins by pemphigus IgG may be (i) part of the mechanism by which sera and IgG induce acantholysis or (ii) a consequence of loss of adhesion (anoikis) and a result of acantholysis [40]. Fas ligand levels are markedly increased in sera from untreated pemphigus patients which is suggesting that Fas/Fas ligand cell death pathway plays a major role in anoikis as seen in pemphigus [41]. Hoechst 33342 staining and RT-PCR analysis showed that apoptosis may be associated with acantholysis of pemphigus lesional epidermis and may play an important role in the pathogenesis of pemphigus [42]. Apoptosis is observed in the perilesional normal appearing tissue suggesting that it is an early phenomenon. Also, the process of apoptosis may cause exacerbation or speeding of the bulla formation [43]. But whether apoptosis is really essential for acantholysis in pemphigus is not clear. Immunofluorescence analysis confirmed that PV IgG autoantibodies promote apoptosis in $\mathrm{HaCaT}$ keratinocytes characterized by caspase- 3 activation, Bcl-2 depletion, and Bax expression [44].

3.3.5. Apoptosis and Epidermolysis Bullosa. Epidermolysis bullosa is a hereditary vesiculobullous disease characterized by blistering of the skin and oral mucosa due to basal keratinocyte fragility [7]. Experiment using cellular model of EBS showed that $\mathrm{HaCaT}$ cells were susceptible to apoptosis by activation of caspases 3 and 8, but not caspase- 9 or caspase12. Moreover TNF- $\alpha$ release and the subsequent activation of the TNF- $\alpha$ receptor by an autocrine/paracrine pathway link protein aggregates to cell death in this keratinocyte EBS cellular model. Also, susceptibility of keratinocytes to caspase-8-mediated apoptosis is increased in mutated K14 because of impairment of the cytoprotective mechanism mediated by K14-TRADD interaction [45].

3.3.6. Apoptosis and Oral Graft-versus-Host Diseases. Graftversus-host disease (GVHD) is characterized by an immunemediated attack by donor immune cells against various recipient host cells and tissues. Although donor and recipient are matched at major histocompatibility loci prior to transplantation, GVHD occurs because differences exist at minor histocompatibility loci. Programmed cell death or apoptosis has been evidenced as major constituent in the pathogenesis of GVHD. Oral involvement may be seen in 33$75 \%$ of patients who develop acute GVHD and in up to $80 \%$ of patients affected by chronic GVHD, making these lesions useful as a highly predictive index for the presence of systemic disease. The oral lesions of GVHD are clinically and histologically lichenoid in nature [46]. CTL mediated apoptotic pathway predominates in GVHD. Initiation can occur either when virus-specific cytotoxic T lymphocytes (CTL) bind to target cells and release granzymes into the cells or when CTL bind and activate the TNF family of receptors, such as the Fasreceptor or TRAIL-receptor [46-48]. Caspases and several intracellular proteases participate in propagating and actuating the apoptotic death signal through a proteolytic cleavage cascade $[49,50]$. Phosphorylation pathways regulate signal transduction cascades [51]. However, experimental murine models of acute GVHD demonstrated that apoptosis can be induced even in the absence of immune cells, suggesting existence of an additional mechanism for target cell injury [52].

3.3.7. Apoptosis and Sjögren's Syndrome. Sjögren's syndrome (SS) is an autoimmune disease characterized by diffuse lymphoid cell infiltrates in the salivary and lacrimal glands, resulting in symptoms of dry mouth and eyes [53]. Three aspects of apoptosis are related to SS: (i) defective apoptosis could lead to lymphoid cell accumulation and chronic inflammation in exocrine glands; (ii) increased apoptosis of epithelial cells might explain the loss of secreting epithelium; and (iii) orderly destruction of cellular components might induce autoantibody production [54]. Apoptosis of the acinar and ductal epithelial cells of the salivary and lacrimal glands may be induced by either CTL through the release perforin and granzyme B or the interaction of Fas ligand, expressed by $\mathrm{T}$ lymphocytes, with Fas on epithelial cells [55]. On the contrary others believed that Fas-induced apoptosis among SS salivary glands is a rare event despite their abundant expression since infiltrating mononuclear cells seem to escape apoptosis, resulting in foci of inflammatory cells [56] $120 \mathrm{kDa}$. $\alpha$-Fodrin is an important autoantigen in the development of SS in animal models and SS patients. Anti-Fas antibodyinduced apoptosis and an increase in the activities of calpain and caspases may be involved in the progression of $\alpha$ fodrin proteolysis and tissue destruction in the development of SS [53]. In vitro analysis demonstrated that apoptosis triggered by anti-Ro and anti-La could be responsible for the impairment of the secretory function in the salivary glands [57].

3.4. Apoptosis and Virus Associated Diseases. Various types of viruses have been involved in the pathogenesis of different oral lesions, the commonest being herpes simplex virus (HSV), human papillomaviruses (HPV), and human immunodeficiency virus (HIV). Virus infected cells can often be recognized and destroyed by apoptotic processes initiated by either CTL or certain cytokines. Many viruses directly induce apoptosis during infection which is at least partially responsible for the various viral pathologies. Many viruses have evolved with multiple distinct mechanisms to block the premature apoptosis of infected cells either facilitating the maintenance of persistent infection or prolonging the survival of lytically infected cells such that the production of progeny virus is maximized [58]. Examples include blockage of apoptosis by viral BCL-2 (vBCL-2) homologues [59], utilization of the phosphatidylinositol 3-kinase-Akt signalling pathway [60], or suppression of inducers of apoptosis such as p53 [61].

HSV infection triggers apoptosis in infected cells. However, proteins synthesized later in infected cells prevent 
apoptotic cell death from ensuing. This apoptotic modulation has a role in the development of herpetic disease [62]. HSV1-induced apoptosis of immature dendritic cells is associated with downregulation of the cellular FLICE-inhibitory protein (c-FLIP) in a proteasome-independent manner [63]. HSV-1 triggers apoptosis in CNS neurons and causes encephalitis in adult subjects [64]. HSV-1 enhances apoptosis via expression of both Fas and Fas ligand on the surface of neonatal neutrophils [65].

High risk HPV oncoproteins E6, E7, and E5 can modulate host mediated apoptosis by inhibiting death receptor signaling and thus regulate the survival of infected cells [66]. HPV E6 proteins inhibit apoptosis in both p53-dependent and p53-independent manners. HPV-18 E6 inhibits Bakinduced apoptosis in differentiating keratinocytes, in which $\mathrm{HPV}$ replicates. It was also suggested that Bak is probably the first naturally occurring target of ubiquitin ligase, E6AP [67].

HIV infection usually leads to progressive immunodeficiency and loss of immune competence associated with declines in both the functionality and the number of CD4+ lymphocytes which in turn is related to various viral and immunological processes. Proposed virus-mediated mechanisms include toxicity caused by accumulation of unintegrated viral DNA, alteration in membrane permeability due to budding of viral particles, and terminal differentiation. Among the immunological mechanisms are killing by specific CTL and signaling through the CD4 molecule, leading to CD4+ lymphocytes apoptosis [68]. Apoptosis in HIV may be stimulated by environmental stress, toxins, and removal of growth factors and may be mediated by one of these death inducing ligands: TNF, FasL, and TRAIL [69]. Many of the proteins that are encoded by the HIV genome have been found to have pro- and/or antiapoptotic qualities. HIV surface glycoprotein, gp120, cross-links CD4 and primes cells for apoptosis. HIV-1 can also induce apoptosis "from within," via expression of certain viral genes, such as tat, nef, vpu, and vpr $[68,69]$.

\subsection{Apoptosis and Benign Entities}

\subsubsection{Apoptosis and Benign Odontogenic Cysts and Tumors}

(1) Apoptosis and Radicular/Apical Cyst. Radicular cysts are the most common cystic lesions of the jaws and represent a periapical inflammatory disease evoked by infected and necrotic dental pulp. Apoptosis was found to be always present in the epithelium of the apical radicular cyst. Higher apoptotic index (AI) and bcl-2 index (bcl-2I) were found in lesions with atrophic compared to hyperplastic epithelium, but the difference was statistically insignificant. However, a positive and significant correlation was found between AI and bcl-2I [70]. Epithelium of radicular and residual cysts showed expression of apoptosis related factors like ssDNA, p53, Bax, Bcl-2, caspase-3, Fas, Fas-L, and Ki-67 antigen suggesting their role in the pathophysiologic activity of periapical inflammatory lesions. Such factors may be affected by the structure of lining epithelium and the degree of inflammatory change since Ki-67 and ssDNA reactivity in RCs with intense inflammatory reactions or with thick lining epithelium were significantly stronger than those RCs with less inflammation or with thin lining [71]. Caspase-3 and Ki-67 expression was significant in hyperplastic epithelium related to intense inflammation, whereas $\mathrm{Bcl}-2$ expression was significantly higher in atrophic epithelium of DCs than RCs suggesting that although they may have different pathogenetic mechanism they have similar biological behavior in the presence of intense inflammatory infiltrate [72].

(2) Apoptosis and Dentigerous Cyst. The epithelial lining of DCs has shown TUNEL, Ki-67, and p53 positivity, but the expression was weak as compared to OKCs indicating that apoptosis is involved in cyst formation and maintaining the regular thickness of the lining epithelium in DCs [73]. Bcl-2 positivity was significantly higher in atrophic epithelium of DCs than that of RCs suggesting that presence of inflammation inhibits the $\mathrm{Bcl}-2$ expression and both cysts have similar biological behavior in the presence of intense inflammatory infiltrate [72] whereas other demonstrated that Bcl-2 expression was almost completely negative in DCs and RCs compared to OKCs [74].

(3) Apoptosis and Odontogenic Keratocyst/Keratocystic Odontogenic Tumor. Keratocystic odontogenic tumor (KCOT), also known as odontogenic keratocyst (OKC), was recently classified as a benign neoplasia due to the aggressive clinical behavior. The proliferation index and apoptotic index were found to be higher and with distinct pattern in both sporadic and nevoid basal cell carcinoma syndrome (NBCCS) KCOTs compared to DCs, reflecting its high cell turnover and reinforcing its classification as an odontogenic tumor [75]. Lack of p16 expression in KCOT compared to strong or moderate p16 expression in RCs and follicular cysts could be related to the increased aggressive behavior, invasiveness, and high frequency of recurrences found in KCOT [76]. Overexpression of cyclin Dl and p53 in syndromic OKCs could be considered as hallmark of a mutated cellular phenotype and more aggressive clinical behaviour compared to sporadic KCOTs which were negative for these oncoproteins [77]. Strong Bcl-2 overexpression in OKCs suggests its role in extension of cell survival leading to the peculiar aggressive growth pattern and can be useful to differentiate OKC from other odontogenic cysts [74]. TUNEL positive cells were observed in the surface layers of OKCs whereas no positivity was seen in the basal or intermediate layers of OKCs and DCs. Also Ki67-positive ratio and p53-positive ratio in the intermediate layer were the highest in OKCs and Bcl-2positive cells were discernible exclusively in the basal layer of OKCs signifying that subsequently OKCs are seen as cystic lesions but not as tumor masses [73]. There is no significant difference in proliferative activity and apoptosis in OKCs associated with or without impacted teeth or between the unilocular or multilocular variants [78]. Survivin immunoexpression was positive in all cases of OKCs while all periapical cysts were negative suggesting its contribution to violent behavior of OKCs [79].

(4) Apoptosis and Ameloblastomas. Ameloblastoma is the most frequently encountered tumor arising from 
odontogenic epithelium and is characterized by a benign but locally invasive behavior with a high risk of recurrence [80].

RT-PCR and IHC showed expression of cytochrome $c$, APAF-1, caspase-9, and AIF in tooth germs and benign and malignant ameloblastomas suggesting that the mitochondriamediated apoptotic pathway has a role in apoptotic cell death of normal and neoplastic odontogenic epithelium and might be involved in oncogenesis, cytodifferentiation, and malignant transformation of odontogenic epithelium [81].

Granular cells, basal cells, desmoplastic ameloblastomas, and ameloblastic carcinomas showed immunoreactivity for the BH3-only proteins (Bid, Bim, Bad, Noxa, and Puma) whereas keratinising cells of acanthomatous ameloblastomas showed no reactivity suggesting that these proteins might be involved in tumor cell differentiation of ameloblastomas [82]. TNF- $\alpha$, TRAIL, and their receptors were also found to be expressed in tooth germs and ameloblastomas proving their role in cytodifferentiation of odontogenic epithelium and tissue structuring of ameloblastomas [83]. Similarly, expression of Fas, FasL, caspase-3, and ssDNA has also been demonstrated in various patterns in tooth germs and benign and malignant ameloblastomas [84].

\subsubsection{Apoptosis and Benign Epithelial Tumor}

(1) Apoptosis and Squamous Papilloma. Oral squamous papilloma (OSP) is a frequent benign tumorous lesion associated with HPV. OSP consists of proliferation of the stratified squamous epithelium resulting in a papillary or verrucous exophytic mass and has got site predilection for the tongue and soft palate, but any surface of the oral cavity can be affected [85, 86]. Carneiro et al. performed immunohistochemical assay on 12 OSP cases utilizing BP53-12 and Pab240 antibodies for p53 protein and found that immunostaining for protein p53 was primarily negative or weakly positive in all cases (in all epithelial layers) except one case which indicates the benign nature of the lesions evidenced by their slow development and less chances of becoming malignant. Intense staining in one slide for both immunomarkers in all the epithelial layers could be attributed to the presence of virus with high oncogenic potential (HPV 16 and 18) or to the presence of coexisting external factors [85].

\subsection{Apoptosis and Premalignant and Malignant Entities}

3.6.1. Apoptosis and Actinic Cheilitis. Actinic cheilitis (AC) is a common condition caused by damage to the lips through exposure to sunlight and has the potential to undergo malignant transformation into squamous cell carcinoma [87]. Overexpression of p53 was found in AC as compared to normal lip and oral mucosa. Bcl-2 expression was higher in $\mathrm{AC}$ than in oral mucosa, but it was significantly reduced as compared with normal lip. Thus, it was suggested that DNA-damaged cells by UV radiation in AC are eliminated by apoptosis. Also, both p53 and bcl-2 play an important role in regulating malignant transformation in $\mathrm{AC}$ [88].

3.6.2. Apoptosis and Oral Leukoplakia. It has been suggested that a change in apoptosis accompanies the onset of invasion in a premalignant lesion of the human oral cavity and oropharynx. AI increased progressively from normal to carcinoma in situ (CIS) but fell in SCC, with maximum apoptotic index (AI): mitotic index (MI) ratio in CIS. The position of apoptotic bodies was localised to the deepest layers in normal and dysplastic epithelia but became generalised in CIS and SCC [89]. Greater expression of oncoproteins MDM2 and Bcl-2 was seen in leukoplakia with altered keratinocyte maturation than OLP case whereas expression of wt-p53 and p21 was higher in OLP compared to leukoplakia cases. In spite of divergent outcome of apoptotic signalling proteins, no significant difference in the number of apoptotic epithelial cells was observed between leukoplakia and OLP cases [90]. Increased mitotic, apoptotic, and $\mathrm{Ki}-$ 67 index indicate unfavourable prognosis of leukoplakia [91]. Abundance of p27 expression in oral leukoplakia may be associated with inhibition of cell proliferation leading to apoptosis of premalignant tumor cells thus preventing tumor progression [92]. It is hypothesised that the antiapoptotic ability of Epstein-Barr virus (EBV) may result in altered expression of apoptosis-associated proteins in oral hairy leukoplakia. Except p53, which appears to be overexpressed, there is only slight alteration in immunoexpression of other apoptosis-associated proteins in OHL [93].

3.6.3. Apoptosis and Oral Squamous Cell Carcinoma. The accumulation of neoplastic cells can occur through enhanced proliferation, diminished cell turnover, or a combination of both processes. Tumor cells from a wide variety of human cancers have been shown to exhibit increased survival and resistance to apoptosis [94]. There are two major ways that could downregulate cancer cell apoptosis: (1) somatic and nonsomatic mutation and loss of expression of proapoptotic molecules and (2) overexpression of apoptosis inhibitory molecules. Mutations within caspase family proteases are not uncommon in malignancies [95]. Caspase-7 proved to be an independent prognosticator and predictor of locoregional recurrence in patients of OSCC [96]. Several alterations that inhibit both the death receptor and mitochondrial pathways via caspases have also been identified including overexpression of IAPs, activation of PI3k or Akt, and posttranslational modifications of caspases [94].

In pathological settings Bcl-2 gene family appears to act synergistically with oncogenes and tumor suppressor genes [97]. Positive Bcl-2 expression is associated with higher tumor grading, higher tumor mitotic index, higher index of atypical mitoses, and microfocal pattern of tumor invasive margins [98]. In OSCC, compared with oral epithelium, there is a decreased $\mathrm{Bcl}-2$ expression, a lowered $\mathrm{Bcl}-2 / \mathrm{Bax}$ ratio, and increased apoptosis. Expression of Bax correlated with histological tumor grading in OSCC [99] whereas others found that expression of Bak may be useful for better characterizing and predicting the prognosis of OSCC [100].

Aberrations of the p53 gene and overexpression of its protein are widely recognized markers of malignancy including OSCC. It was found that there is a negative correlation of p53 immunoexpression with histologic grade of differentiation. In patients without neck node metastases, p53 showed a strong correlation with survival [101]. On the contrary, 
another study showed that expression of p53 protein did not behave as a marker of prognostic value in patients with OSCC [102].

As cancer is characterized by uncontrolled cell proliferation, markers of proliferation, such as Ki67 and PCNA, have been studied extensively in neoplastic lesions. Expression of both p53 and Ki67 correlated significantly with the histopathological stage of the tumor. However, expression of p53 was not correlated with that of Ki67 [103].

HPV infection and/or p53 mutations are implicated in carcinogenesis of oral SCC but are not mutually exclusive events. Moreover, decrease in apoptosis is more closely related to p53 mutation than HPV infection [104].

Survivin has got a versatile role in modulating cell division and apoptosis in cancer. Low survivin expression correlated statistically significantly with better survival rates but not with age, sex, tumor size, the presence of lymph node, or distant metastases [105]. Survivin was found to be preferentially expressed in nonadvanced, nonmetastatic, and chemotherapy-sensitive OSCC [106]. On the contrary, increased expression of survivin was found in high-grade tumors suggesting its contribution to apoptosis resistance in response to therapy [107]. Also significant correlation between survivin expression, lymph node metastasis, and proliferation was revealed but not with differentiation, microvessel density, or TNM staging [108].

Upregulation of FasL and downregulation of Fas have been shown to occur in oral carcinogenesis, possibly contributing to both cancer cell survival and evasion of the host immune assault through induction of apoptosis in $\mathrm{T}$ cells that would normally kill tumor cells [109]. Moreover, it has been found that Fas is expressed in low quantities in OSCC whereas FasL expression correlates negatively with degree of differentiation and apoptosis in OSCC [110]. Thus, various proteins (apoptotic and antiapoptotic) have got a significant bearing in initiation, progression, and prognosis of OSCC.

3.7. Apoptosis and Salivary Gland Tumors. Salivary gland tumors (SGT) are relatively uncommon neoplasms with widely variable histopathologic and biologic characteristics. P53/bcl-2 immunostaining reactivity could be helpful in demonstrating SGT behavior in terms of progression and extent of invasion since all malignant salivary gland tumors were positive whereas $70 \%$ of pleomorphic adenomas (PA) showed negative expression [111]. Similarly, Nordkvist et al. found p53 overexpression in $20 \%$ of the malignant SGT, with the highest prevalence in PLGA, squamous cell carcinoma, and CaXPA and the lowest in adenoid cystic carcinoma and acinic cell carcinoma but none of the benign PA [112]. Expression of p53 in malignant tumors bore no correlation to local recurrence, metastatic disease, or survival of the patients [113]. Bcl-2 protein expression was found virtually in all benign and malignant SGT suggesting its role in the development and proliferation of these tumors via inhibition of apoptosis [114]. p53 expression, apoptotic index, and bcl2 protein are potential prognostic parameters in salivary gland adenoid cystic carcinoma [115]. Frequency of apoptosis is inversely related to bcl-2 but not to Bax expression in SGT [116]. Survival probability of patients who demonstrated positive staining for c-erbB-2 or TUNEL or both was lower than those with negative staining [117].

\section{Conclusion}

Understanding the mechanistic machinery of apoptosis is crucial because it influences both health and disease, being initiated by various physiologic and pathologic stimuli. Moreover, the extensive involvement of apoptosis in the pathogenesis of various oral diseases and meticulous knowledge about it prompt it to therapeutic intervention at different checkpoints. This review is an attempt to provide an update on the crucial role and importance of apoptosis in various oral pathologies.

\section{Conflict of Interests}

The authors declare that there is no conflict of interests regarding the publication of this paper.

\section{References}

[1] A. Lawen, "Apoptosis: an introduction," BioEssays, vol. 25, no. 9, pp. 888-896, 2003.

[2] J. F. Kerr, A. H. Wyllie, and A. R. Currie, "Apoptosis: a basic biological phenomenon with wide-ranging implications in tissue kinetics," British Journal of Cancer, vol. 26, no. 4, pp. 239-257, 1972.

[3] A. Strasser, L. O’Connor, and V. M. Dixit, "Apoptosis signaling," Annual Review of Biochemistry, vol. 69, pp. 217-245, 2000.

[4] N. Singh, "Apoptosis in health and disease and modulation of apoptosis for therapy: an overview," Indian Journal of Clinical Biochemistry, vol. 22, no. 2, pp. 6-16, 2007.

[5] T.-J. Fan, L.-H. Han, R.-S. Cong, and J. Liang, "Caspase family proteases and apoptosis," Acta Biochimica et Biophysica Sinica, vol. 37, no. 11, pp. 719-727, 2005.

[6] P. C. Ashe and M. D. Berry, "Apoptotic signaling cascades," Progress in Neuro-Psychopharmacology and Biological Psychiatry, vol. 27, no. 2, pp. 199-214, 2003.

[7] L. L. Loro, O. K. Vintermyr, and A. C. Johannessen, "Apoptosis in normal and diseased oral tissues," Oral Diseases, vol. 11, no. 5, pp. 274-287, 2005.

[8] H. Jafarzadeh, M. Sanatkhani, and N. Mohtasham, "Oral pyogenic granuloma: a review," Journal of Oral Science, vol. 48, no. 4, pp. 167-175, 2006.

[9] T. Nakamura, "Apoptosis and expression of Bax/Bcl-2 proteins in pyogenic granuloma: a comparative study with granulation tissue and capillary hemangioma," Journal of Cutaneous Pathology, vol. 27, no. 8, pp. 400-405, 2000.

[10] K. Yuan, L.-Y. C. Wing, and M. T. Lin, "Pathogenetic roles of angiogenic factors in pyogenic granulomas in pregnancy are modulated by female sex hormones," Journal of Periodontology, vol. 73, no. 7, pp. 701-708, 2002.

[11] K. Yuan and M. T. Lin, "The roles of vascular endothelial growth factor and angiopoietin-2 in the regression of pregnancy pyogenic granuloma," Oral Diseases, vol. 10, no. 3, pp. 179-185, 2004.

[12] F. R. Amaral, V. F. Bernardes, A. P. Duarte et al., "Quantitative expression analysis of apoptotic/antiapoptotic genes and association with immunolocalization of BAX and BCL-2 in peripheral 
and central giant cell lesions of the jaws," Tumor Biology, vol. 32, no. 5, pp. 997-1003, 2011.

[13] J. Pammer, W. Weninger, H. Hulla, P. Mazal, and R. Horvat, "Expression of regulatory apoptotic proteins in peripheral giant cell granulomas and lesions containing osteoclast-like giant cells," Journal of Oral Pathology and Medicine, vol. 27, no. 6, pp. 267-271, 1998.

[14] C. Scully and S. Porter, "Oral mucosal disease: recurrent aphthous stomatitis," British Journal of Oral and Maxillofacial Surgery, vol. 46, no. 3, pp. 198-206, 2008.

[15] T. Honma, T. Saito, and Y. Fujioka, "Possible role of apoptotic cells of the oral epithelium in the pathogenesis of aphthous ulceration," Oral Surgery Oral Medicine and Oral Pathology, vol. 59, no. 4, pp. 379-387, 1985.

[16] J. F. N. Pinto Rodrigues, C. T. Fujiyama Oshima, A. P. Ribeiro Paiotti, M. Franco, S. S. Miki Ihara, and D. A. Ribeiro, "Expression of apoptosis regulatory proteins p53, bcl-2 and bax in recurrent aphthous ulceration," Journal of the European Academy of Dermatology and Venereology, vol. 26, no. 10, pp. 1247-1251, 2012.

[17] J. Gamonal, A. Bascones, A. Acevedo, E. Blanco, and A. Silva, "Apoptosis in chronic adult periodontitis analyzed by in situ DNA breaks, electron microscopy, and immunohistochemistry," Journal of Periodontology, vol. 72, no. 4, pp. 517-525, 2001.

[18] F. Jarnbring, E. Somogyi, J. Dalton, A. Gustafsson, and B. Klinge, "Quantitative assessment of apoptotic and proliferative gingival keratinocytes in oral and sulcular epithelium in patients with gingivitis and periodontitis," Journal of Clinical Periodontology, vol. 29, no. 12, pp. 1065-1071, 2002.

[19] P. Kelk, A. Johansson, R. Claesson, L. Hänström, and S. Kalfas, "Caspase 1 involvement in human monocyte lysis induced by Actinobacillus actinomycetemcomitans leukotoxin," Infection and Immunity, vol. 71, no. 8, pp. 4448-4455, 2003.

[20] P. G. Stathopoulou, J. C. Galicia, M. R. Benakanakere, C. A. Garcia, J. Potempa, and D. F. Kinane, "Porphyromonas gingivalis induce apoptosis in human gingival epithelial cells through a gingipain-dependent mechanism," BMC Microbiology, vol. 9, article 107, 2009.

[21] T. Murata, K. Yaegaki, W. Qian et al., "Hydrogen sulfide induces apoptosis in epithelial cells derived from human gingival," Journal of Breath Research, vol. 2, no. 1, Article ID 017007, 2008.

[22] J. C. Galicia, M. R. Benakanakere, P. G. Stathopoulou, and D. F. Kinane, "Neutrophils rescue gingival epithelial cells from bacterial-induced apoptosis," Journal of Leukocyte Biology, vol. 86, no. 1, pp. 181-186, 2009.

[23] A. Kantarci, P. Augustin, E. Firatli et al., "Apoptosis in gingival overgrowth tissues," Journal of Dental Research, vol. 86, no. 9, pp. 888-892, 2007.

[24] V. A. Murrah and E. P. Gilchrist, "Assessment of apoptosis in oral lichen planus," Oral Surgery, Oral Medicine, Oral Pathology, Oral Radiology, and Endodontics, vol. 82, no. 2, pp. 209-210, 1996.

[25] M. R. Roopashree, R. V. Gondhalekar, M. C. Shashikanth, J. George, S. H. Thippeswamy, and A. Shukla, "Pathogenesis of oral lichen planus: a review," Journal of Oral Pathology and Medicine, vol. 39, no. 10, pp. 729-734, 2010.

[26] R. Mattila and S. Syrjänen, "Caspase cascade pathways in apoptosis of oral lichen planus," Oral Surgery, Oral Medicine, Oral Pathology, Oral Radiology and Endodontology, vol. 110, no. 5, pp. 618-623, 2010.

[27] J. M. C. Brant, A. C. Vasconcelos, and L. V. Rodrigues, "Role of apoptosis in erosive and reticular oral lichen planus exhibiting variable epithelial thickness," Brazilian Dental Journal, vol. 19, no. 3, pp. 179-185, 2008.

[28] B. K. Bloor, F. K. Malik, E. W. Odell, and P. R. Morgan, "Quantitative assessment of apoptosis in oral lichen planus," Oral Surgery, Oral Medicine, Oral Pathology, Oral Radiology, and Endodontics, vol. 88, no. 2, pp. 187-195, 1999.

[29] F. A. C. G. D. Sousa, T. C. Paradella, Y. R. Carvalho, and L. E. B. Rosa, "Immunohistochemical expression of PCNA, p53, bax and bcl-2 in oral lichen planus and epithelial dysplasia," Journal of oral science, vol. 51, no. 1, pp. 117-121, 2009.

[30] C. Bascones-Ilundain, M. A. Gonzalez-Moles, G. EsparzaGómez, J. A. Gil-Montoya, and A. Bascones-Martínez, "Importance of apoptotic mechanisms in inflammatory infiltrate of oral lichen planus lesions," Anticancer Research, vol. 26, no. 1, pp. 357-362, 2006.

[31] V. N. Pimentel, L. S. de Matos, T. C. B. Soares et al., "Perforin and granzyme B involvement in oral lesions of lichen planus and chronic GVHD," Journal of Oral Pathology and Medicine, vol. 39, no. 10, pp. 741-746, 2010.

[32] L. R. Oliveira and S. Zucoloto, "Erythema multiforme minor: a revision," The American Journal of Infectious Diseases, vol. 4, no. 4, pp. 224-231, 2008.

[33] E. Chrysomali, F. Lozada-Nur, N. P. Dekker, S. I. Papanicolaou, and J. A. Regezi, "Apoptosis in oral erythema multiforme," Oral Surgery, Oral Medicine, Oral Pathology, Oral Radiology, and Endodontics, vol. 83, no. 2, pp. 272-280, 1997.

[34] R. Abe, T. Shimizu, A. Shibaki, H. Nakamura, H. Watanabe, and H. Shimizu, "Toxic epidermal necrolysis and Stevens-Johnson syndrome are induced by soluble fas ligand," The American Journal of Pathology, vol. 162, no. 5, pp. 1515-1520, 2003.

[35] S. Inachi, H. Mizutani, and M. Shimizu, "Epidermal apoptotic cell death in erythema multiforme and Stevens-Johnson syndrome: contribution of perforin-positive cell infiltration," Archives of Dermatology, vol. 133, no. 7, pp. 845-849, 1997.

[36] A. K. Vaishnaw, E. Toubi, S. Ohsako et al., "The spectrum of apoptotic defects and clinical manifestations, including systemic lupus erythematosus, in humans with Cd95 (Fas/Apo1) mutations," Arthritis and Rheumatism, vol. 42, no. 9, pp. 1833$1842,1999$.

[37] S. White and A. Rosen, "Apoptosis in systemic lupus erythematosus," Current Opinion in Rheumatology, vol. 15, no. 5, pp. 557-562, 2003.

[38] D. Shah, S. Sah, and S. K. Nath, "Interaction between glutathione and apoptosis in systemic lupus erythematosus," Autoimmunity Reviews, vol. 12, no. 7, pp. 741-751, 2013.

[39] S. Rajagopalan, E. C. Somers, R. D. Brook et al., "Endothelial cell apoptosis in systemic lupus erythematosus: a common pathway for abnormal vascular function and thrombosis propensity," Blood, vol. 103, no. 10, pp. 3677-3683, 2004.

[40] M. Bektas, P. Jolly, and D. S. Rubenstein, "Apoptotic pathways in pemphigus," Dermatology Research and Practice, vol. 2010, Article ID 456841, 2010.

[41] M. Puviani, A. Marconi, E. Cozzani, and C. Pincelli, "Fas ligand in pemphigus sera induces keratinocyte apoptosis through the activation of caspase-8," Journal of Investigative Dermatology, vol. 120, no. 1, pp. 164-167, 2003.

[42] B. H. Roh, K. U. Whang, M. K. Cho, Y. L. Park, J. S. Lee, and C. W. Lee, "A study of apoptosis in pemphigus vulgaris," Korean Journal of Dermatology, vol. 45, no. 7, pp. 650-658, 2007.

[43] P. Deyhimi and P. Tavakoli, "Study of apoptosis in oral pemphigus vulgaris using immunohistochemical marker Bax and 
TUNEL technique," Journal of Oral Pathology and Medicine, vol. 42, no. 5, pp. 409-414, 2013.

[44] B. Pelacho, C. Natal, A. España, I. Sánchez-Carpintero, M. J. Iraburu, and M. J. López-Zabalza, "Pemphigus vulgaris autoantibodies induce apoptosis in $\mathrm{HaCaT}$ keratinocytes," FEBS Letters, vol. 566, no. 1-3, pp. 6-10, 2004.

[45] K. Yoneda, T. Furukawa, Y.-J. Zheng et al., "An autocrine/ paracrine loop linking keratin 14 aggregates to tumor necrosis dactor $\alpha$-mediated cytotoxicity in a keratinocyte model of epidermolysis bullosa simplex," Journal of Biological Chemistry, vol. 279, no. 8, pp. 7296-7303, 2004.

[46] P. P. Sedghizadeh, C. M. Allen, K. E. Anderson, D. H. Kim, J. R. Kalmar, and J. C. Lang, "Oral graft-versus-host disease and programmed cell death: pathogenetic and clinical correlates," Oral Surgery, Oral Medicine, Oral Pathology, Oral Radiology, and Endodontics, vol. 97, no. 4, pp. 491-498, 2004.

[47] T. A. Graubert, J. H. Russell, and T. J. Ley, “The role of granzyme $\mathrm{B}$ in murine models of acute graft-versus-host disease and graft rejection," Blood, vol. 87, no. 4, pp. 1232-1237, 1996.

[48] N. Zavazava and D. Kabelitz, "Alloreactivity and apoptosis in graft rejection and transplantation tolerance," Journal of Leukocyte Biology, vol. 68, no. 2, pp. 167-174, 2000.

[49] A. Sarin, E. K. Haddad, and P. A. Henkart, "Caspase dependence of target cell damage induced by cytotoxic lymphocytes," Journal of Immunology, vol. 161, no. 6, pp. 2810-2816, 1998.

[50] M. Enari, H. Hug, and S. Nagata, "Involvement of an ICE-like protease in Fas-mediated apoptosis," Nature, vol. 375, no. 6526, pp. 78-81, 1995.

[51] T. H. Holmstrom and J. E. Eriksson, "Phophorylation based signaling in Fas receptor mediated apoptosis," Critical Reviews in Immunology, vol. 20, pp. 121-152, 2000.

[52] Y. H. Yoo, A. C. Gilliam, D. Whitaker-Menezes, R. Korngold, and G. F. Murphy, "Experimental induction and ultrastructural characterization of apoptosis in marine acute cutaneous graftversus-host disease," Archives of Dermatological Research, vol. 289, no. 7, pp. 389-398, 1997.

[53] Y. Hayashi, K. Yayagi, and N. Haneji, "Involvement of apoptotic protease cascade for tissue destruction in Sjögren's syndrome," Archivum Immunologiae et Therapiae Experimentalis, vol. 48, no. 5, pp. 399-403, 2000.

[54] A. I. Bolstad and R. Jonsson, “The role of apoptosis in Sjögren's syndrome," Annals of Internal Medicine, vol. 149, no. 1, pp. 2529, 1998.

[55] P. Manganelli and P. Fietta, "Apoptosis and Sjögren syndrome," Seminars in Arthritis and Rheumatism, vol. 33, no. 1, pp. 49-65, 2003.

[56] M. Ohlsson, K. Skarstein, A. I. Bolstad, A. C. Johannessen, and R. Jonsson, "Fas-induced apoptosis is a rare event in Sjögren's syndrome," Laboratory Investigation, vol. 81, no. 1, pp. 95-105, 2001.

[57] M. Sisto, S. Lisi, D. Lofrumento, M. D’Amore, P. Scagliusi, and V. Mitolo, "Autoantibodies from Sjögren's syndrome trigger apoptosis in salivary gland cell line," Annals of the New York Academy of Sciences, vol. 1108, pp. 418-425, 2007.

[58] L. S. Young, C. W. Dawson, and A. G. Eliopoulos, "Viruses and apoptosis," British Medical Bulletin, vol. 53, no. 3, pp. 509-521, 1997.

[59] B. M. Polster, J. Pevsner, and J. M. Hardwick, "Viral Bcl-2 homologs and their role in virus replication and associated diseases," Biochimica et Biophysica Acta, vol. 1644, no. 2-3, pp. 211-227, 2004.
[60] S. Cooray, "The pivotal role of phosphatidylinositol 3-kinaseAkt signal transduction in virus survival," Journal of General Virology, vol. 85, no. 5, pp. 1065-1076, 2004.

[61] J. G. Teodoro and P. E. Branton, "Regulation of apoptosis by viral gene products," Journal of Virology, vol. 71, no. 3, pp. 1739-1746, 1997.

[62] M. L. Nguyen and J. A. Blaho, "Apoptosis during herpes simplex virus infection," Advances in Virus Research, vol. 69, pp. 67-97, 2006.

[63] A. Kather, M. J. Raftery, G. Devi-Rao et al., "Herpes simplex virus type 1 (HSV-1)-induced apoptosis in human dendritic cells as a result of downregulation of cellular FLICE-inhibitory protein and reduced expression of HSV-1 antiapoptotic latencyassociated transcript sequences," Journal of Virology, vol. 84, no. 2, pp. 1034-1046, 2010.

[64] L. Aurelian, "HSV-induced apoptosis in herpes encephalitis," Current Topics in Microbiology and Immunology, vol. 289, pp. 79-111, 2005.

[65] J. Ennaciri, J. Menezes, F. Proulx, and B. J. Toledano, "Induction of apoptosis by herpes simplex virus-1 in neonatal, but not adult, neutrophils," Pediatric Research, vol. 59, no. 1, pp. 7-12, 2006.

[66] A. M. Fuentes-González, A. Contreras-Paredes, J. ManzoMerino, and M. Lizano, "The modulation of apoptosis by oncogenic viruses,” Virology Journal, vol. 10, article 182, 2013.

[67] M. Thomas and L. Banks, "Inhibition of Bak-induced apoptosis by HPV-18 E6," Oncogene, vol. 17, no. 23, pp. 2943-2954, 1998.

[68] M. Roshal, Y. Zhu, and V. Planelles, "Apoptosis in AIDS," Apoptosis, vol. 6, no. 1-2, pp. 103-116, 2001.

[69] N. W. Cummins and A. D. Badley, "Mechanisms of HIVassociated lymphocyte apoptosis: 2010," Cell Death and Disease, vol. 1, no. 11, article e99, 2010.

[70] A. M. Loyola, S. V. Cardoso, G. S. Lisa et al., "Apoptosis in epithelial cells of apical radicular cysts," International Endodontic Journal, vol. 38, no. 7, pp. 465-469, 2005.

[71] T. Suzuki, H. Kumamoto, K. Kunimori, and K. Ooya, "Immunohistochemical analysis of apoptosis-related factors in lining epithelium of radicular cysts," Journal of Oral Pathology and Medicine, vol. 34, no. 1, pp. 46-52, 2005.

[72] C. A. Martins, E. R. C. Rivero, R. M. Dufloth, C. P. Figueiredo, and D. S. C. Vieira, "Immunohistochemical detection of factors related to cellular proliferation and apoptosis in radicular and dentigerous cysts," Journal of Endodontics, vol. 37, no. 1, pp. 3639, 2011.

[73] E. Kichi, Y. Enokiya, T. Muramatsu et al., "Cell proliferation, apoptosis and apoptosis-related factors in odontogenic keratocysts and in dentigerous cysts," Journal of Oral Pathology and Medicine, vol. 34, no. 5, pp. 280-286, 2005.

[74] A. Piattelli, M. Fioroni, and C. Rubini, "Differentiation of odontogenic keratocysts from other odontogenic cysts by the expression of bcl-2 immunoreactivity," Oral Oncology, vol. 34, no. 5, pp. 404-407, 1998.

[75] G. C. P. Mateus, G. H. D. S. P. Lanza, P. H. R. de Moura, H. A. de Marigo, and M. C. R. Horta, "Cell proliferation and apoptosis in keratocystic odontogenic tumors," Medicina Oral, Patologia Oral y Cirugia Bucal, vol. 13, no. 11, pp. E697-E702, 2008.

[76] L. Artese, A. Piattelli, C. Rubini et al., "p16 expression in odontogenic tumors," Tumori, vol. 94, no. 5, pp. 718-723, 2008.

[77] L. Lo Muzio, S. Staibano, G. Pannone et al., "Expression of cell cycle and apoptosis-related proteins in sporadic odontogenic keratocysts and odontogenic keratocysts associated with the nevoid basal cell carcinoma syndrome," Journal of Dental Research, vol. 78, no. 7, pp. 1345-1353, 1999. 
[78] D. K. Kim, S. G. Ahn, J. Kim, and J. H. Yoon, "Comparative Ki-67 expression and apoptosis in the odontogenic keratocyst associated with or without an impacted tooth in addition to unilocular and multilocular varieties," Yonsei Medical Journal, vol. 44, no. 5, pp. 841-846, 2003.

[79] M. Andric, B. Dozic, B. Popovic et al., "Survivin expression in odontogenic keratocysts and correlation with cytomegalovirus infection," Oral Diseases, vol. 16, no. 2, pp. 156-159, 2010.

[80] I. R. H. Kramer, J. J. Pindborg, and M. Shear, WHO Histological Typing of Odontogenic Tumours, Springer, Berlin, Germany, 1992.

[81] H. Kumamoto and K. Ooya, "Detection of mitochondriamediated apoptosis signaling molecules in ameloblastomas," Journal of Oral Pathology and Medicine, vol. 34, no. 9, pp. 565572, 2005.

[82] H. Kumamoto and K. Ooya, "Immunohistochemical detection of BH3-only proteins in ameloblastic tumors," Oral Diseases, vol. 14, no. 6, pp. 550-555, 2008.

[83] H. Kumamoto and K. Ooya, "Expression of tumor necrosis factor $\alpha$, TNF-related apoptosis-inducing ligand, and their associated molecules in ameloblastomas," Journal of Oral Pathology and Medicine, vol. 34, no. 5, pp. 287-294, 2005.

[84] H. Kumamoto, K. Kimi, and K. Ooya, "Immunohistochemical analysis of apoptosis-related factors (Fas, Fas ligand, caspase-3 and single-stranded DNA) in ameloblastomas," Journal of Oral Pathology and Medicine, vol. 30, no. 10, pp. 596-602, 2001.

[85] T. E. Carneiro, S. A. Marinho, F. D. Verli, A. T. M. Mesquita, N. L. Lima, and J. L. Miranda, "Oral squamous papilloma: clinical, histologic and immunohistochemical analyses," Journal of oral science, vol. 51, no. 3, pp. 367-372, 2009.

[86] T. Yamaguchi, M. Shindoh, A. Amemiya et al., "Detection of human papillomavirus type 2 related sequence in oral papilloma," Analytical Cellular Pathology, vol. 16, no. 3, pp. 125$130,1998$.

[87] J. H. Main and M. Pavone, "Actinic cheilitis and carcinoma of the lip," Journal of Canadian Dental Association, vol. 60, no. 2, pp. 113-116, 1994.

[88] A. Martínez, U. Brethauer, I. G. Rojas et al., "Expression of apoptotic and cell proliferation regulatory proteins in actinic cheilitis," Journal of Oral Pathology and Medicine, vol. 34, no. 5, pp. 257-262, 2005.

[89] M. A. Birchall, C. M. Winterford, D. J. Allan, and B. V. Harmon, "Apoptosis in normal epithelium, premalignant and malignant lesions of the oropharynx and oral cavity: a preliminary study," European Journal of Cancer B, vol. 31, no. 6, pp. 380-383, 1995.

[90] N. Tanda, S. Mori, K. Saito, K. Ikawa, and S. Sakamoto, "Expression of apoptotic signaling proteins in leukoplakia and oral lichen planus: quantitative and topographical studies," Journal of Oral Pathology and Medicine, vol. 29, no. 8, pp. 385393, 2000.

[91] G. Kövesi and B. Szende, "Progression of the leukoplakia is associated to changes in apoptotic and mitotic index as well as in p53 and Ki-67 expression," Oncology, vol. 46, no. 4, pp. 333338, 2002.

[92] H. Tsuzuki, S. Fujieda, H. Sunaga, N. Narita, M. Tokuriki, and H. Saito, "Expression of p27 and apoptosis in oral leukoplakia," Anticancer Research, vol. 23, no. 2, pp. 1265-1270, 2003.

[93] E. Chrysomali, J. S. Greenspan, N. Dekker, D. Greenspan, and J. A. Regezi, "Apoptosis-associated proteins in oral hairy leukoplakia," Oral Diseases, vol. 2, no. 4, pp. 279-284, 1996.

[94] S. H. Kaufmann and G. J. Gores, "Apoptosis in cancer: cause and cure," BioEssays, vol. 22, pp. 1007-1017, 2000.
[95] S. Ghavami, M. Hashemi, S. R. Ande et al., "Apoptosis and cancer: mutations within caspase genes," Journal of Medical Genetics, vol. 46, no. 8, pp. 497-510, 2009.

[96] C. M. Coutinho-Camillo, S. V. Lourenço, I. N. Nishimoto, L. P. Kowalski, and F. A. Soares, "Caspase expression in oral squamous cell carcinoma," Head and Neck, vol. 33, no. 8, pp. 1191-1198, 2011.

[97] P. J. Polverini and J. E. Nör, "Apoptosis and predisposition to oral cancer," Critical Reviews in Oral Biology and Medicine, vol. 10, no. 2, pp. 139-152, 1999.

[98] M. Sulkowska, W. Famulski, S. Sulkowski et al., "Correlation between Bcl-2 protein expression and some clinicopathological features of oral squamous cell carcinoma," Polish Journal of Pathology, vol. 54, no. 1, pp. 49-52, 2003.

[99] L. L. Loro, O. K. Vintermyr, P. G. Liavaag, R. Jonsson, and A. C. Johannessen, "Oral squamous cell carcinoma is associated with decreased bcl-2/bax expression ratio and increased apoptosis," Human Pathology, vol. 30, no. 9, pp. 1097-1105, 1999.

[100] M. Baltaziak, M. Koda, M. Barwijuk-Machała et al., "The role of Bak expression in apoptosis of the oral squamous cell carcinoma (OSCC) and metastases to lymph nodes (LNMs)," Annales Academiae Medicae Bialostocensis, vol. 49, pp. 14-15, 2004.

[101] J. C. de Vicente, L. M. J. Gutiérrez, A. H. Zapatero, M. F. F. Forcelledo, G. Hernández-Vallejo, and J. S. López Arranz, "Prognostic significance of p53 expression in oral squamous cell carcinoma without neck node metastases," Head and Neck, vol. 26, no. 1, pp. 22-30, 2004.

[102] M. A. Gonzalez-Moles, P. Galindo, J. Gutierrez-Fernandez et al., "P53 protein expression in oral squamous cell carcinoma: survival analysis," Anticancer Research, vol. 21, no. 4, pp. 28892894, 2001

[103] S. Kannan, G. Jagadeesh Chandran, K. Raveendran Pillai et al., "Expression of p53 in leukoplakia and squamous cell carcinoma of the oral mucosa: correlation with expression of Ki67," Journal of Clinical Pathology, vol. 49, no. 3, pp. M170-M175, 1996.

[104] J. Y. Koh, N. P. Cho, G. Kong, J. D. Lee, and K. Yoon, “p53 mutations and human papillomavirus DNA in oral squamous cell carcinoma: correlation with apoptosis," British Journal of Cancer, vol. 78, no. 3, pp. 354-359, 1998.

[105] L. Lo Muzio, G. Pannone, S. Staibano et al., "Survivin expression in oral squamous cell carcinoma," British Journal of Cancer, vol. 89, no. 12, pp. 2244-2248, 2003.

[106] Y. Jinbu, K. Tsukinoki, N. Miyagi et al., "Expression of survivin in oral squamous cell carcinoma," Oral Med Pathol, vol. 11, pp. 41-44, 2006.

[107] C. Jane, A. V. Nerurkar, N. V. Shirsat, R. B. Deshpande, A. D. Amrapurkar, and F. R. Karjodkar, "Increased survivin expression in high-grade oral squamous cell carcinoma: a study in Indian tobacco chewers," Journal of Oral Pathology and Medicine, vol. 35, no. 10, pp. 595-601, 2006.

[108] Y.-H. Kim, S.-M. Kim, Y.-K. Kim, S.-P. Hong, M.-J. Kim, and H. Myoung, "Evaluation of survivin as a prognostic marker in oral squamous cell carcinoma," Journal of Oral Pathology and Medicine, vol. 39, no. 5, pp. 368-375, 2010.

[109] N. G. Nikitakis, J. J. Sauk, and S. I. Papanicolaou, “The role of apoptosis in oral disease: mechanisms, aberrations in neoplastic, autoimmune, infectious, hematologic, and developmental diseases, and therapeutic opportunities," Oral Surgery, Oral Medicine, Oral Pathology, Oral Radiology, and Endodontics, vol. 97, no. 4, pp. 476-490, 2004.

[110] L. L. Loro, O. K. Vintermyr, A. C. Johannessen, P. G. Liavaag, and R. Jonsson, "Suppression of Fas receptor and negative 
correlation of Fas ligand with differentiation and apoptosis in oral squamous cell carcinoma," Journal of Oral Pathology and Medicine, vol. 28, no. 2, pp. 82-87, 1999.

[111] N. H. Al-Rawi, H. Omer, and S. Al Kawas, "Immunohistochemical analysis of P53 and bcl-2 in benign and malignant salivary glands tumors," Journal of Oral Pathology and Medicine, vol. 39, no. 1, pp. 48-55, 2010.

[112] A. Nordkvist, E. Röijer, G. Bang et al., "Expression and mutation patterns of p53 in benign and malignant salivary gland tumors," International Journal of Oncology, vol. 16, no. 3, pp. 477-483, 2000.

[113] V. J. Kärjä, K. J. Syrjänen, A.-K. Kurvinen, and S. M. Syrjänen, "Expression and mutations of p53 in salivary gland tumours," Journal of Oral Pathology and Medicine, vol. 26, no. 5, pp. 217223, 1997.

[114] M. Yanezez, I. Roa, M. García, G. Ibacache, and M. Villaseca, "Gene Bcl-2 protein expression in salivary gland tumors," Revista Medica de Chile, vol. 127, no. 2, pp. 139-142, 1999.

[115] L. Jia, R. L. Esguerra, X. Tang et al., "Prognostic value of apoptosis and apoptosis-associated proteins in salivary gland adenoid cystic carcinoma," Pathology International, vol. 54, no. 4, pp. 217-223, 2004.

[116] Y. Soini, U. Tormanen, and P. Paakko, "Apoptosis is inversely related to bcl-2 but not to bax expression in salivary gland tumours," Histopathology, vol. 32, no. 1, pp. 28-34, 1998.

[117] R. M. Nagler, H. Kerner, S. Ben-Eliezer, I. Minkov, and O. BenItzhak, "Prognostic role of apoptotic, Bcl-2, c-erbB-2 and p53 tumor markers in salivary gland malignancies," Oncology, vol. 64, no. 4, pp. 389-398, 2003. 


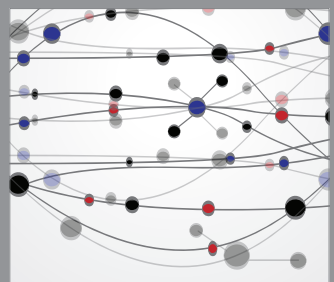

The Scientific World Journal
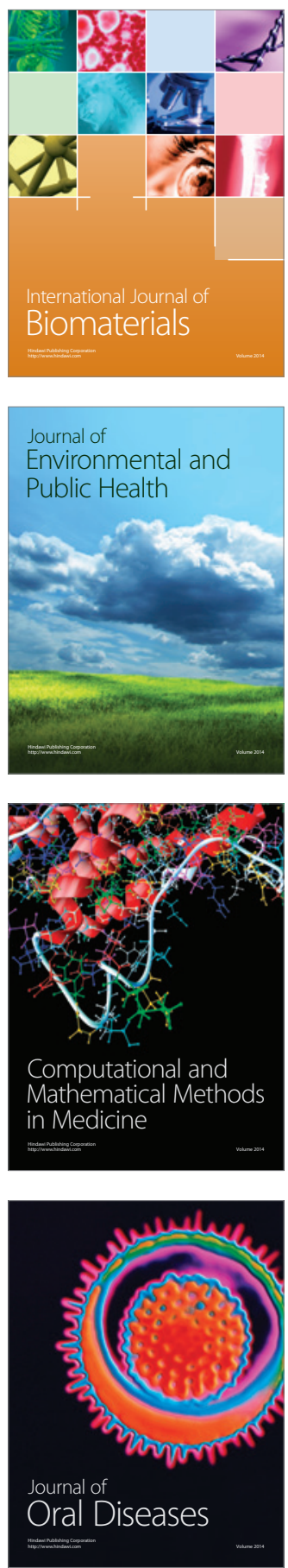
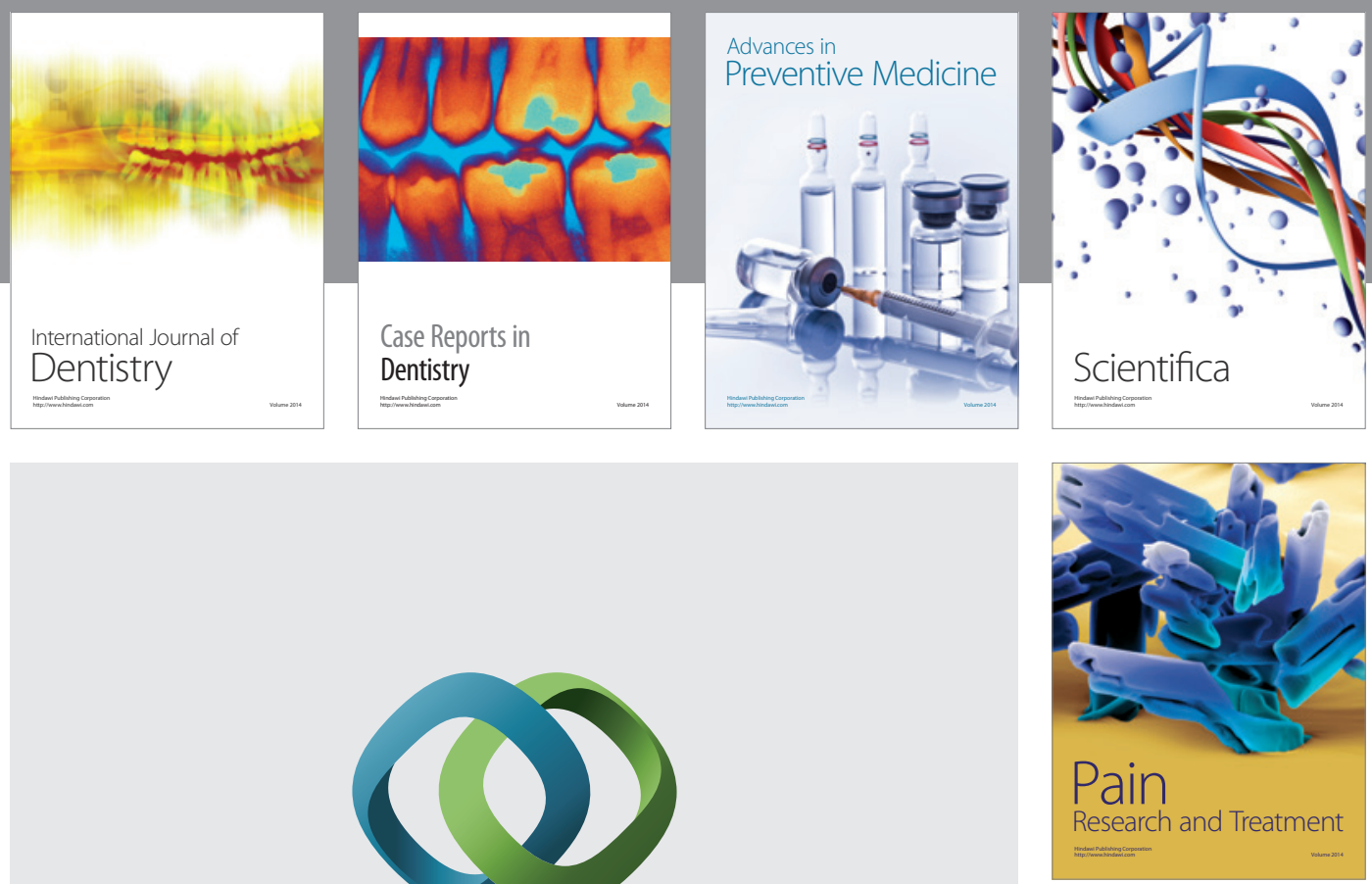

\section{Hindawi}

Submit your manuscripts at

http://www.hindawi.com
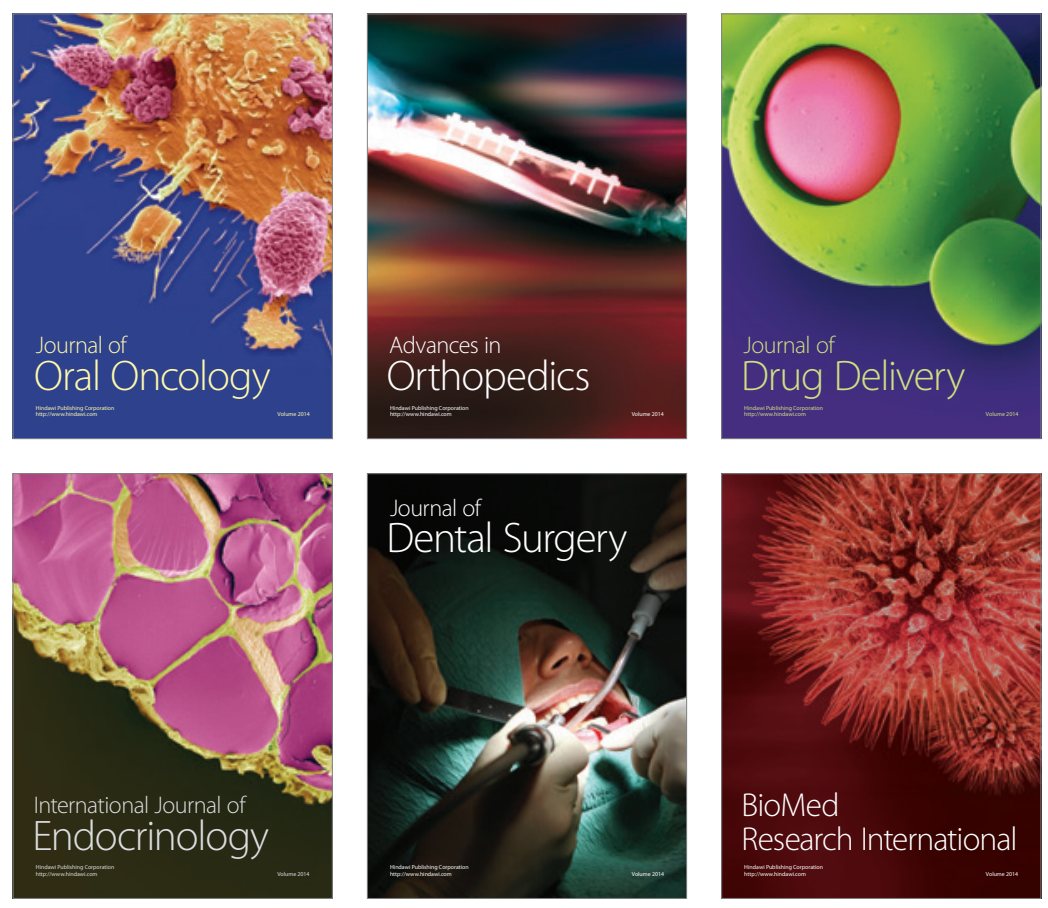

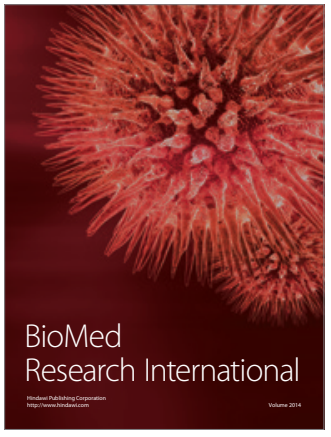

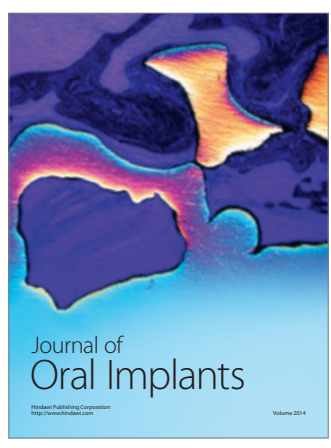
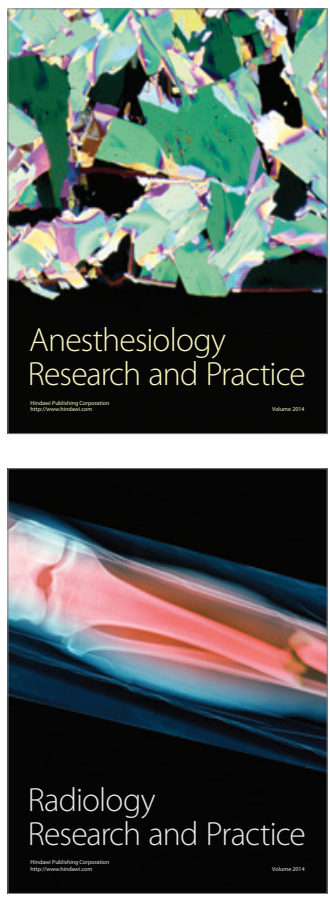